\title{
Nonlinear Stefan problem with convective boundary condition in Storm's materials*
}

\author{
Adriana C. BRIOZZO ${ }^{(1)(2)}$ - María F. NATALE ${ }^{(1)}$ \\ (1) Depto. de Matemática, F.C.E.,Univ. Austral, Paraguay 1950, \\ S2000FZF Rosario, ARGENTINA. \\ (2) CONICET \\ E-mail: abriozzo@austral.edu.ar,fnatale@austral.edu.ar
}

\begin{abstract}
We consider a nonlinear one-dimensional Stefan problem for a semi-infinite material $x>0$, with phase change temperature $T_{f}$. We assume that the heat capacity and the thermal conductivity satisfy a Storm's condition and we assume a convective boundary condition at the fixed face $x=0$. An unique explicit solution of similarity type is obtained. Moreover, asymptotic behavior of the solution when $h \rightarrow+\infty$ is studied.
\end{abstract}

Key Words: Stefan problem, free boundary problem, phase-change process, similarity solution

AMS Subject Classification: 35R35, 80A22, 35C05.

\section{Introduction}

As in $[4,8,10]$ we consider the following one phase nonlinear unidimensional Stefan problem for a semi-infinite material $x>0$, with phase change temperature $T_{f}$

$$
\begin{gathered}
s(T) \frac{\partial T}{\partial t}=\frac{\partial}{\partial x}\left[k(T) \frac{\partial T}{\partial x}\right], 0<x<X(t), t>0 \\
k(T(0, t)) \frac{\partial T}{\partial x}(0, t)=\frac{h}{\sqrt{t}}\left[T(0, t)-T_{m}\right], h>0, t>0, \\
T(X(t), t)=T_{f},
\end{gathered}
$$

*All correspondence concerning this paper should be sent to first author. 


$$
\begin{gathered}
k\left(T_{f}\right) \frac{\partial T}{\partial x}(X(t), t)=\alpha \dot{X}(t), t>0, \\
X(0)=0
\end{gathered}
$$

where the positive constant $\alpha$ is $\rho L, L$ is the latent heat of fusion of the medium, $\rho$ is the density (assumed constant), $T_{m}$ is the temperature of the medium $T_{m}<T(0, t)<T_{f}$ and $h_{0}$ is the positive heat transfer coefficient.

We assume that the metal exhibits nonlinear thermal characteristics such that the heat capacity $c_{p}(T)>0$ and the thermal conductivity $k(T)>0$ satisfy a Storm's condition $[1,2,5,6,7,9]$

$$
\frac{\frac{d}{d T}\left(\sqrt{\frac{s(T)}{k(T)}}\right)}{s(T)}=\lambda=\text { const. }>0,
$$

where $s(T)=\rho c_{p}(T)$.

Condition (6) was originally obtained by [9] in an investigation of heat conduction in simple monoatomic metals. There, the validity of the approximation (6) was examined for aluminium, silver, sodium, cadium, zinc, copper and lead.

In [7] the free boundary problem (1) - (6) (fusion case) for the particular case $k(T)=$ $\rho c /(a+b T)^{2}$ and $s(T)=\rho c=$ constant was studied. The explicit solution of this problem was obtained through the unique solution of an integral equation with time as a parameter. A similar case with the constant temperature at the fixed face $x=0$ was also studied.

In [2] two nonlinear Stefan problems analogous to (1) - (5) with phase change temperature $T_{f}$ and the Storm's condition (6) are considered. In one case a heat flux boundary condition of the type $q(t)=\frac{q_{0}}{\sqrt{t}}$ and in the other case a temperature boundary condition $T=T_{s}<T_{f}$ at the fixed face $x=0$ are assumed. Solutions of similarity type are obtained in both cases and the equivalence of the two problems is demonstrated.

The goal of this paper is to determine the temperature $T=T(x, t)$ and the position of the phase change boundary at time $t, X=X(t)$, which satisfy the problem (1) - (6). In the section 2 we show how to find a unique solution of the similarity type for this problem. In Section 3 we study the asymptotic behavior when $h \rightarrow+\infty$. We prove that the solutions $T=T_{h}(x, t), X=X_{h}(t)$ of $(1)-(5)$ converges to the solution $T=T_{\infty}(x, t), X=X_{\infty}(t)$ of an analogous Stefan problem with temperature condition $T(0, t)=T_{m}$ when $h \rightarrow+\infty$.

\section{Existence and uniqueness of the solution to the Ste- fan problem with convective boundary condition on the fixed face}

We consider the problem (1) - (6) and we propose a similarity type solution given by $[2,3,4]$

$$
T(x, t)=\Phi(\xi), \xi=\frac{x}{X(t)}
$$


where

$$
X(t)=\sqrt{2 \gamma t}, t>0
$$

is the free boundary and $\gamma$ is assumed a positive constant to be determined.

Then we have that the problem $(1)-(5)$ is equivalent to

$$
\begin{gathered}
k(\Phi) \Phi^{\prime \prime}(\xi)+k^{\prime}(\Phi) \Phi^{\prime 2}(\xi)+\gamma s(\Phi) \Phi^{\prime}(\xi) \xi=0 \quad, 0<\xi<1, \\
k(\Phi(0)) \Phi^{\prime}(0)=h \sqrt{2 \gamma}\left[\Phi(0)-T_{m}\right], \\
\phi(1)=T_{f}, \\
k(\Phi(1)) \Phi^{\prime}(1)=\alpha \gamma .
\end{gathered}
$$

If we define

$$
y(\xi)=\sqrt{\frac{k}{s}(\Phi(\xi))},
$$

then a parametrization of the Storm condition (6) is

$$
s(\Phi)=-\frac{1}{\lambda y^{2}} \frac{d y}{d \Phi}, \quad k(\Phi)=-\frac{1}{\lambda} \frac{d y}{d \Phi}
$$

and then we have that the following problem is equivalent to (9) - (12)

$$
\begin{gathered}
\frac{d^{2} y}{d \xi^{2}}+\frac{\gamma \xi}{y^{2}} \frac{d y}{d \xi}=0 \quad, \quad 0<\xi<1, \\
y^{\prime}(0)=-\lambda h \sqrt{2 \gamma}\left[P\left(y^{2}(0)\right)-T_{m}\right], \\
y^{\prime}(1)=-\alpha \lambda \gamma, \\
y(1)=y_{1}=\sqrt{\frac{k\left(T_{f}\right)}{s\left(T_{f}\right)}} .
\end{gathered}
$$

where $P$ is the inverse function of the decreasing function $\frac{k}{s}$.

Lemma 1 A parametric solution to the problem (15) - (18) is given by

$$
\begin{gathered}
\xi=\varphi_{1}(u)=\frac{F_{u_{0}}(u)}{F_{u_{0}}\left(u_{1}\right)}, \\
y=\varphi_{2}(u)=\frac{\sqrt{\gamma} \sqrt{\frac{\pi}{2}}\left[\operatorname{erf}\left(\frac{u}{\sqrt{2}}\right)-g\left(\frac{u_{0}}{\sqrt{2}}, \frac{1}{\sqrt{\pi}}\right)\right]}{F_{u_{0}}\left(u_{1}\right)},
\end{gathered}
$$

for

$$
u_{0} \leq u \leq u_{1}
$$


where the function $F_{u_{0}}=F_{u_{0}}(u)$ was defined in [2] as follow $F_{u_{0}}(u)=\exp \left(-\frac{u^{2}}{2}\right)+u\left(\int_{u_{0}}^{u} \exp \left(-\frac{z^{2}}{2}\right) d z-\frac{\exp \left(-\frac{u_{0}^{2}}{2}\right)}{u_{0}}\right)=\sqrt{\frac{\pi}{2}} u\left[g\left(\frac{u}{\sqrt{2}}, \frac{1}{\sqrt{\pi}}\right)-g\left(\frac{u_{0}}{\sqrt{2}}, \frac{1}{\sqrt{\pi}}\right)\right], u \geq u_{0}$ where $u_{0}, u_{1}$ are the parameter values which verify that $\xi=\varphi_{1}\left(u_{0}\right)=0$ and $\xi=\varphi_{1}\left(u_{1}\right)=1$,

$$
g(x, p)=\operatorname{erf}(x)+p \frac{\exp \left(-x^{2}\right)}{x}, p>0, x>0
$$

and

$$
\operatorname{erf}(x)=\frac{2}{\sqrt{\pi}} \int_{0}^{x} \exp \left(-z^{2}\right) d z, x>0 .
$$

The unknowns $\gamma, u_{0}$ and $u_{1}$ must verify the following system of equations

$$
\begin{gathered}
u_{0}=\sqrt{2} \lambda h\left[P\left(\frac{\gamma \exp \left(-u_{0}^{2}\right)}{\left[u_{0} F_{u_{0}}\left(u_{1}\right)\right]^{2}}\right)-T_{m}\right], \\
\sqrt{\gamma}=\frac{\exp \left(-\frac{u_{1}^{2}}{2}\right)}{\sqrt{\frac{\pi}{2}} \alpha \lambda\left[g\left(\frac{u_{0}}{\sqrt{2}}, \frac{1}{\sqrt{\pi}}\right)-\operatorname{erf}\left(\frac{u_{1}}{\sqrt{2}}\right)\right]} \\
y_{1}=\frac{-\exp \left(-\frac{u_{1}^{2}}{2}\right)}{\alpha \lambda F_{u_{0}}\left(u_{1}\right)}
\end{gathered}
$$

Proof. A parametric solution of (15) was deduced in [4] and it is given by

$$
\begin{aligned}
& \xi=\varphi_{1}(u)=C_{2}\left(\exp \left(-\frac{u^{2}}{2}\right)+u\left(\int_{0}^{u} \exp \left(-\frac{x^{2}}{2}\right) d x+C_{1}\right)\right) \\
& y=\varphi_{2}(u)=\sqrt{\gamma} C_{2}\left(\int_{0}^{u} \exp \left(-\frac{x^{2}}{2}\right) d x+C_{1}\right), u>0
\end{aligned}
$$

where $C_{1}$ and $C_{2}$ are integration constants to be determined.

We choose $u_{0}$ and $u_{1}$ be such that $\varphi_{1}\left(u_{0}\right)=0$ and $\varphi_{1}\left(u_{1}\right)=1$, we obtain that

$$
\begin{gathered}
C_{1}=-\frac{\exp \left(-\frac{u_{0}^{2}}{2}\right)}{u_{0}}-\int_{0}^{u_{0}} \exp \left(-\frac{x^{2}}{2}\right) d x \\
C_{2}=\left\{\exp \left(-\frac{u_{1}^{2}}{2}\right)+u_{1}\left(-\frac{\exp \left(-\frac{u_{0}^{2}}{2}\right)}{u_{0}}+\int_{u_{0}}^{u_{1}} \exp \left(-\frac{x^{2}}{2}\right) d x\right)\right\}^{-1} .
\end{gathered}
$$


Then, we have

$$
\xi=\varphi_{1}(u)=\frac{\exp \left(-\frac{u^{2}}{2}\right)+u\left(\int_{u_{0}}^{u} \exp \left(-\frac{x^{2}}{2}\right) d x-\frac{\exp \left(-\frac{u_{0}^{2}}{2}\right)}{u_{0}}\right)}{\exp \left(-\frac{u_{1}^{2}}{2}\right)+u_{1}\left(-\frac{\exp \left(-\frac{u_{0}^{2}}{2}\right)}{u_{0}}+\int_{u_{0}}^{u_{1}} \exp \left(-\frac{x^{2}}{2}\right) d x\right)}, u_{0} \leq u \leq u_{1}
$$

and

$$
y=\varphi_{2}(u)=\frac{\sqrt{\gamma}\left\{-\frac{\exp \left(-\frac{u_{0}^{2}}{2}\right)}{u_{0}}+\int_{u_{0}}^{u} \exp \left(-\frac{x^{2}}{2}\right) d x\right\}}{\exp \left(-\frac{u_{1}^{2}}{2}\right)+u_{1}\left(-\frac{\exp \left(-\frac{u_{0}^{2}}{2}\right)}{u_{0}}+\int_{u_{0}}^{u_{1}} \exp \left(-\frac{x^{2}}{2}\right) d x\right)}, u_{0} \leq u \leq u_{1}
$$

that is $(15)-(18)$.

Next we prove that the unknowns $u_{0}, u_{1}$ and $\gamma$ must satisfy (22) - (24). From (29) and (30) we have

$$
y^{\prime}(\xi)=\frac{\varphi_{2}^{\prime}(u)}{\varphi_{1}^{\prime}(u)}=\frac{\sqrt{\gamma} \exp \left(-\frac{u^{2}}{2}\right)}{\int_{u_{0}}^{u} \exp \left(-\frac{x^{2}}{2}\right) d x-\frac{\exp \left(-\frac{u_{0}^{2}}{2}\right)}{u_{0}}}
$$

then

$$
y^{\prime}(0)=-\sqrt{\gamma} u_{0}
$$

and taking into account that

$$
y(0)=\frac{-\sqrt{\gamma} \exp \left(-\frac{u_{0}^{2}}{2}\right)}{u_{0} F_{u_{0}}\left(u_{1}\right)}
$$

and from (16) we have (22).

Analogously we have

$$
y^{\prime}(1)=\frac{\varphi_{2}^{\prime}\left(u_{1}\right)}{\varphi_{1}^{\prime}\left(u_{1}\right)}=\frac{\sqrt{\gamma} \exp \left(-\frac{u_{1}^{2}}{2}\right)}{\int_{u_{0}}^{u_{1}} \exp \left(-\frac{x^{2}}{2}\right) d x-\frac{\exp \left(-\frac{u_{0}^{2}}{2}\right)}{u_{0}}}
$$

and by (17) we have

$$
\frac{\sqrt{\gamma} \exp \left(-\frac{u_{1}^{2}}{2}\right)}{\int_{u_{0}}^{u_{1}} \exp \left(-\frac{x^{2}}{2}\right) d x-\frac{\exp \left(-\frac{u_{0}^{2}}{2}\right)}{u_{0}}}=-\alpha \lambda \gamma
$$


that is $(23)$.

Last, we have

$$
y(1)=\varphi_{2}\left(u_{1}\right)=\frac{\sqrt{\gamma}\left\{-\frac{\exp \left(-\frac{u_{0}^{2}}{2}\right)}{u_{0}}+\int_{u_{0}}^{u_{1}} \exp \left(-\frac{x^{2}}{2}\right) d x\right\}}{\exp \left(-\frac{u_{1}^{2}}{2}\right)+u_{1}\left(-\frac{\exp \left(-\frac{u_{0}^{2}}{2}\right)}{u_{0}}+\int_{u_{0}}^{u_{1}} \exp \left(-\frac{x^{2}}{2}\right) d x\right)}
$$

and taking into account (18) and (23) we obtain (24).

Next we want to find $u_{0}, u_{1}$ and $\gamma$ the solutions to the equations $(22)-(24)$. We can rewrite the system $(22)-(24)$ as follow

$$
\begin{gathered}
P^{-1}\left(\frac{u_{0}}{\sqrt{2} h \lambda}+T_{m}\right)=\frac{\gamma \exp \left(-u_{0}^{2}\right)}{\left[u_{0} F_{u_{0}}\left(u_{1}\right)\right]^{2}} \\
\sqrt{\gamma}=\frac{\exp \left(-\frac{u_{1}^{2}}{2}\right)}{\alpha \lambda \sqrt{\frac{\pi}{2}}\left[g\left(\frac{u_{0}}{\sqrt{2}}, \frac{1}{\sqrt{\pi}}\right)-\operatorname{erf}\left(\frac{u_{1}}{\sqrt{2}}\right)\right]} \\
M\left(u_{1}\right)=g\left(\frac{u_{0}}{\sqrt{2}}, \frac{1}{\sqrt{\pi}}\right)
\end{gathered}
$$

where

$$
M(x)=g\left(\frac{x}{\sqrt{2}}, \frac{1}{\sqrt{\pi}}\left(\frac{1}{\alpha \lambda y_{1}}+1\right)\right)
$$

Lemma 2 The real function $F_{u_{0}}$ and $M$ satisfy the following properties:

$$
\begin{gathered}
F_{u_{0}}\left(u_{0}\right)=0, F(+\infty)=-\infty \\
F_{u_{0}}^{\prime}(x)=\frac{\sqrt{\pi}}{2}\left\{\operatorname{erf}\left(\frac{x}{\sqrt{2}}\right)-g\left(\frac{u_{0}}{\sqrt{2}}, \frac{1}{\sqrt{\pi}}\right)\right\}<0 \\
M(0)=+\infty, \quad M(+\infty)=1 \text { and } M^{\prime}(x)<0 .
\end{gathered}
$$

Proof. See [1] and [2].

\section{Lemma 3 (Existence of the solution)}

There exists a solution of the system (36) - (38) given by

$$
\tilde{u}_{1}=M^{-1}\left(g\left(\frac{\tilde{u}_{0}}{\sqrt{2}}, \frac{1}{\sqrt{\pi}}\right)\right)
$$




$$
\tilde{\gamma}=\frac{\exp \left(-\tilde{u}_{1}^{2}\right)}{\alpha^{2} \lambda^{2}\left(\frac{\exp \left(-\frac{\tilde{u}_{0}^{2}}{2}\right)}{\tilde{u}_{0}}-\int_{\tilde{u}_{0}}^{\tilde{u}_{1}} \exp \left(-\frac{x^{2}}{2}\right) d x\right)^{2}}
$$

where $\tilde{u}_{0}$ is a solution of

$$
P^{-1}\left(\frac{u_{0}}{\sqrt{2} h \lambda}+T_{m}\right)=\frac{\gamma \exp \left(-u_{0}^{2}\right)}{\left[u_{0} F_{u_{0}}\left(M^{-1}\left(g\left(\frac{u_{0}}{\sqrt{2}}, \frac{1}{\sqrt{\pi}}\right)\right)\right)\right]^{2}} .
$$

Proof. Because $M$ is a decreasing function there exists the inverse function $M^{-1}$ and from (38) for each $u_{0}$ there exists a unique $u_{1}$ given by

$$
u_{1}\left(u_{0}\right)=M^{-1}\left(g\left(\frac{u_{0}}{\sqrt{2}}, \frac{1}{\sqrt{\pi}}\right)\right) .
$$

If we replace (46) in (37) and (36) we have

$$
\gamma\left(u_{0}\right)=\frac{\exp \left(-u_{1}^{2}\left(u_{0}\right)\right)}{\alpha^{2} \lambda^{2}\left(\frac{\exp \left(-\frac{u_{0}^{2}}{2}\right)}{u_{0}}-\int_{u_{0}}^{u_{1}\left(u_{0}\right)} \exp \left(-\frac{x^{2}}{2}\right) d x\right)^{2}}
$$

and

$$
P^{-1}\left(\frac{u_{0}}{\sqrt{2} h \lambda}+T_{m}\right)=\frac{\gamma\left(u_{0}\right) \exp \left(-u_{0}^{2}\right)}{\left[u_{0} F_{u_{0}}\left(u_{1}\left(u_{0}\right)\right)\right]^{2}}
$$

We define the function

$$
G\left(u_{0}\right):=P^{-1}\left(\frac{u_{0}}{\sqrt{2} h \lambda}+T_{m}\right)
$$

which satisfies $G(0)=\frac{k}{s}\left(T_{m}\right)$ and $G^{\prime}\left(u_{0}\right)<0$, and let

$$
H\left(u_{0}\right):=\frac{\gamma\left(u_{0}\right) \exp \left(-u_{0}^{2}\right)}{\left[u_{0} F_{u_{0}}\left(u_{1}\left(u_{0}\right)\right)\right]^{2}} .
$$

From (24), (46) and (47) it follows that

$$
H\left(u_{0}\right)=\frac{2 y_{1}^{2} \exp \left(-u_{0}^{2}\right)}{u_{0}^{2} \pi\left[\operatorname{erf}\left(\frac{M^{-1}\left(g\left(\frac{u_{0}}{\sqrt{2}}, \frac{1}{\sqrt{\pi}}\right)\right)}{\sqrt{2}}\right)-g\left(\frac{u_{0}}{\sqrt{2}}, \frac{1}{\sqrt{\pi}}\right)\right]^{2}},
$$

$H(0)=y_{1}^{2}, H(+\infty)=+\infty$ and $H\left(u_{0}\right) \geq y_{1}^{2}, \forall u_{0} \geq 0$.

Since $T_{m}<T_{f}$ we conclude $G(0)=\frac{k}{s}\left(T_{m}\right)>\frac{k}{s}\left(T_{f}\right)=y_{1}^{2}=H(0)$. Taking into account that the properties of $G$ and $H$ there exists $\tilde{u}_{0}<u_{0}^{*}=\left(T_{f}-T_{m}\right) \sqrt{2} h \lambda$ which satisfies (48). Then by (46) and (47) we complete the solution $\tilde{u}_{1}=u_{1}\left(\tilde{u}_{0}\right)$ and $\tilde{\gamma}_{=} \gamma\left(\tilde{u}_{0}\right)$ to the system $(36)-(38)$. 


\section{Lemma 4 (Uniqueness of the solution)}

The solution $\left(\tilde{u}_{0}, \tilde{u}_{1}, \tilde{\gamma}\right)$ to the system $(22)-(24)$ is unique.

Proof. Suppose the assertion of the lemma is false. That is there exist two solutions $\left(\tilde{u}_{0}, \tilde{u}_{1}, \tilde{\gamma}\right)$ and $\left(u_{0}^{*}, u_{1}^{*}, \gamma^{*}\right)$ to $(22)-(24)$.

We assume that $\tilde{u}_{0}<u_{0}^{*}$, then by (19) we have

$$
\xi=\frac{F_{u_{0}^{*}}(u)}{F_{u_{0}^{*}}\left(u_{1}^{*}\right)}=\frac{F_{\tilde{u}_{0}}(u)}{F_{\tilde{u}_{0}}\left(\tilde{u}_{1}\right)}, \quad \text { for } \quad u_{0}^{*} \leq u \leq \min \left(\tilde{u}_{1}, u_{1}^{*}\right) .
$$

For $u=u_{0}^{*}$ we have

$$
0=\frac{F_{u_{0}^{*}}\left(u_{0}^{*}\right)}{F_{u_{0}^{*}}\left(u_{1}^{*}\right)}=\frac{F_{\tilde{u}_{0}}\left(u_{0}^{*}\right)}{F_{\tilde{u}_{0}}\left(\tilde{u}_{1}\right)}
$$

then $F_{\tilde{u}_{0}}\left(u_{0}^{*}\right)=0$. This is a contradiction because $F_{\tilde{u}_{0}}\left(u_{0}^{*}\right)=0$ if and only if $u=\tilde{u}_{0}$.

Theorem 5 The problem (1) - (6) has a similarity type solution given by

$$
T(x, t)=P\left(\left(\varphi_{2}\left(\varphi_{1}^{-1}(x / X(t))\right)\right)^{2}\right), \quad 0<x<X(t)
$$

where

$$
X(t)=\sqrt{2 \tilde{\gamma} t}, \quad t>0
$$

is the free boundary,

$$
\begin{gathered}
\varphi_{1}(u)=\frac{F_{\tilde{u}_{0}}(u)}{F_{\tilde{u}_{0}}\left(\tilde{u}_{1}\right)}, \\
\varphi_{2}(u)=\frac{\sqrt{\tilde{\gamma}} \sqrt{\frac{\pi}{2}}\left[\operatorname{erf}\left(\frac{u}{\sqrt{2}}\right)-g\left(\frac{\tilde{u}_{0}}{\sqrt{2}}, \frac{1}{\sqrt{\pi}}\right)\right]}{F_{\tilde{u}_{0}}\left(\tilde{u}_{1}\right)},
\end{gathered}
$$

$\left(\tilde{u}_{0}, \tilde{u}_{1}, \tilde{\gamma}\right)$ is the unique solution of $(22)-(24)$ and $P=\left(\frac{k}{s}\right)^{-1}$ is the inverse function of the function $\frac{k}{s}$.

Proof. Fixed the data: $\alpha, \lambda, h, T_{f}$ of the problem $(1)-(6)$, we obtain the solutions of the equations $(22)-(24)$ given by (43), (44) and $\tilde{u}_{0}$ is the solution of (45).

Next, we obtain $\varphi_{1}$ and $\varphi_{2}$ given by $(53),(54)$ respectively and the free boundary is $X(t)=\sqrt{2 \tilde{\gamma} t}$. Taking into account that $\varphi_{1}$ is an increasing function we determine $\varphi_{1}^{-1}\left(\frac{x}{X(t)}\right)$. Finally, we invert the relation (13) and from (7) we obtain (51).

Remark 1 Si $T(0, t)=T_{s}$ is constant, the convective condition (2) at the fixed face $x=0$ of the problem (1) - (6) becomes a Neumann boundary condition given by

$$
k(T(0, t)) \frac{\partial T}{\partial x}(0, t)=\frac{q_{0}}{\sqrt{t}}
$$

with

$$
q_{0}=h\left[T_{s}-T_{m}\right]
$$

The Stefan problem (1) - (6) with the condition (55) instead (2) was studied in [2]. 


\section{Asymptotic behavior of the solution when $h \rightarrow+\infty$}

Let $h>0$ and $T=T_{h}(x, t), X=X_{h}(t)$ denote the solution to the problem (1) - (6) given by $(51)-(54)$. We will study the behavior of this solution when the transfer coefficient $h \rightarrow+\infty$. We will prove that $T_{h}, X_{h}$ converges to the solution $T_{\infty}, X_{\infty}$ of the following parabolic free boundary problem:

$$
\begin{gathered}
s(T) \frac{\partial T}{\partial t}=\frac{\partial}{\partial x}\left[k(T) \frac{\partial T}{\partial x}\right] \quad, 0<x<X(t), t>0 \\
T(0, t)=T_{m}, t>0 \\
T(X(t), t)=T_{f}, t>0 \\
k\left(T_{f}\right) \frac{\partial T}{\partial x}(X(t), t)=\alpha \dot{X}(t), t>0 \\
X(0)=0
\end{gathered}
$$

with the Storm's condition

$$
\frac{\frac{d}{d T}\left(\sqrt{\frac{s(T)}{k(T)}}\right)}{s(T)}=\lambda .
$$

The problem (56) - (61) was studied in [2]. The solution is given by

$$
T_{\infty}(x, t)=P\left(\left(\varphi_{2 \infty}\left(\varphi_{1 \infty}^{-1}\left(x / X_{\infty}(t)\right)\right)\right)^{2}\right)
$$

$$
X_{\infty}(t)=\sqrt{2 \gamma_{\infty} t}
$$

where

$$
\begin{gathered}
\varphi_{1 \infty}(u)=\frac{F_{v_{0}}(u)}{F_{v_{0}}\left(v_{1}\right)}, \\
\varphi_{2 \infty}(u)=\frac{\sqrt{\gamma_{\infty}} \sqrt{\frac{\pi}{2}}\left[\operatorname{erf}\left(\frac{u}{\sqrt{2}}\right)-g\left(\frac{v_{0}}{\sqrt{2}}, \frac{1}{\sqrt{\pi}}\right)\right]}{F_{v_{0}}\left(v_{1}\right)}
\end{gathered}
$$

with $v_{0} \leq u \leq v_{1}$. The parameters $v_{0}, v_{1}$ and $\gamma_{\infty}$ satisfy the following equations

$$
\begin{gathered}
y_{1}=\sqrt{\gamma_{\infty}} \frac{F_{v_{0}}\left(v_{1}\right)-\exp \left(-\frac{v_{1}^{2}}{2}\right)}{v_{1} F_{v_{0}}\left(v_{1}\right)} \\
\sqrt{\gamma_{\infty}}=\frac{v_{1} y_{1}}{1+\alpha \lambda y_{1}} \\
\frac{k}{s}\left(T_{m}\right)=y_{0}=-\sqrt{\gamma_{\infty}} \frac{\exp \left(-\frac{v_{0}^{2}}{2}\right)}{v_{0} F_{v_{0}}\left(v_{1}\right)}
\end{gathered}
$$


which are equivalent to

$$
\begin{gathered}
\frac{k}{s}\left(T_{m}\right)=H\left(v_{0}\right)=\frac{2 y_{1}^{2} \exp \left(-v_{0}^{2}\right)}{v_{0}^{2} \pi\left[\operatorname{erf}\left(\frac{M^{-1}\left(g\left(\frac{v_{0}}{\sqrt{2}}, \frac{1}{\sqrt{\pi}}\right)\right)}{\sqrt{2}}\right)-g\left(\frac{v_{0}}{\sqrt{2}}, \frac{1}{\sqrt{\pi}}\right)\right]^{2}} \\
\sqrt{\gamma_{\infty}}=\frac{\exp \left(-\frac{v_{1}^{2}}{2}\right)}{\alpha \lambda \sqrt{\frac{\pi}{2}}\left[g\left(\frac{v_{0}}{\sqrt{2}}, \frac{1}{\sqrt{\pi}}\right)-\operatorname{erf}\left(\frac{v_{1}}{\sqrt{2}}\right)\right]} \\
v_{1}=M^{-1}\left(g\left(\frac{v_{0}}{\sqrt{2}}, \frac{1}{\sqrt{\pi}}\right)\right) .
\end{gathered}
$$

For simplicity of notation, we wright $\left(u_{0 h}, u_{1 h}, \gamma_{h}\right)$ instead of $\left(\tilde{u}_{0 h}, \tilde{u}_{1 h}, \tilde{\gamma}_{h}\right)$ which is the solution of $(36)-(38)$. Firstly we will prove that $\left(u_{0 h}, u_{1 h}, \gamma_{h}\right)$ converges to $\left(v_{0}, v_{1}, \gamma_{\infty}\right)$ when $h \rightarrow+\infty$. The proof of this statement is based on the following lemma:

Lemma 6 The sequences $\left\{u_{0 h}\right\},\left\{u_{1 h}\right\}$ and $\left\{\gamma_{h}\right\}$ are increasing and bounded. Moreover

$$
\lim _{h \rightarrow+\infty} u_{0 h}=v_{0}, \quad \lim _{h \rightarrow+\infty} u_{1 h}=v_{1}, \quad \text { and } \quad \lim _{h \rightarrow+\infty} \gamma_{h}=\gamma_{\infty}
$$

Proof. From properties of function $G=G_{h}(x)=P^{-1}\left(\frac{x}{\sqrt{2} h \lambda}+T_{m}\right)$ we have

a) $h_{1} \leq h_{2} \Rightarrow G_{h_{1}}(x) \leq G_{h_{2}}(x), \quad \forall x \geq 0$

b) $G_{h}(x) \leq \frac{k}{s}\left(T_{m}\right), \quad \forall x \geq 0, \quad h>0$.

We consider $h_{1}<h_{2}$, if $u_{0 h_{1}}$ and $u_{0 h_{2}}$ are the solutions of $G_{h_{1}}(x)=H(x)$ and $G_{h_{2}}(x)=$ $H(x)$ respectively, by a) and properties of function $H$ we have that $u_{0 h_{1}}<u_{0 h_{2}}$. Moreover from b) results $u_{0 h} \leq v_{0}$ for all $h>0$. Then, $\left\{u_{0 h}\right\}$ is an increasing bounded sequence and there exists $\tilde{u}_{0}$ such that

$$
\lim _{h \rightarrow+\infty} u_{0 h}=\tilde{u_{0}} .
$$

Letting $h \rightarrow+\infty$ on $G_{h}\left(u_{0 h}\right)=H\left(u_{0 h}\right)$ yields $\frac{k}{s}\left(T_{m}\right)=H\left(\tilde{u_{0}}\right)$. By uniqueness of the solution of (69) results $\tilde{u}_{0}=v_{0}$.

From (38) we have

$$
u_{1 h}=M^{-1}\left(g\left(\frac{u_{0 h}}{\sqrt{2}}, \frac{1}{\sqrt{\pi}}\right)\right)
$$

Because $\left\{u_{0 h}\right\}$ is increasing, $M$ and $g$ are decreasing functions we have that the sequence $\left\{u_{1 h}\right\}$ is increasing. Moreover taking into account $u_{0 h} \leq v_{0}$ and (71) follows

$$
u_{1 h}=M^{-1}\left(g\left(\frac{u_{0 h}}{\sqrt{2}}, \frac{1}{\sqrt{\pi}}\right)\right) \leq M^{-1}\left(g\left(\frac{v_{0}}{\sqrt{2}}, \frac{1}{\sqrt{\pi}}\right)\right)=v_{1}
$$


for all $h>0$.

By (72) we obtain

$$
\lim _{h \rightarrow+\infty} u_{1 h}=\lim _{h \rightarrow+\infty} M^{-1}\left(g\left(\frac{u_{0 h}}{\sqrt{2}}, \frac{1}{\sqrt{\pi}}\right)\right)=M^{-1}\left(g\left(\frac{v_{0}}{\sqrt{2}}, \frac{1}{\sqrt{\pi}}\right)\right)=v_{1} .
$$

Finally, letting $h \rightarrow+\infty$ in (37) we have

$$
\lim _{h \rightarrow+\infty} \gamma_{h}=\gamma_{\infty}
$$

It follows easily of (37) and (38) that $\sqrt{\gamma_{h}}=\frac{u_{1 h} y_{1}}{1+\alpha \lambda y_{1}}$. Taking into account $u_{1 h} \leq v_{1}$ we have

$$
\sqrt{\gamma_{h}}=\frac{u_{1 h} y_{1}}{1+\alpha \lambda y_{1}} \leq \frac{v_{1} y_{1}}{1+\alpha \lambda y_{1}}=\sqrt{\gamma_{\infty}} \quad \forall h>0
$$

Corollary 7 For each $t>0$, the sequence $\left\{X_{h}(t)\right\}$ is monotonically increasing and $\lim _{h \rightarrow+\infty} X_{h}(t)=$ $X_{\infty}(t)$.

We can now define an extension $\tilde{T}_{h}=\tilde{T}_{h}(x, t) \in C^{1}\left[0, X_{\infty}(t)\right]$ of $T_{h}(x, t)$ as follows

$$
\tilde{T}_{h}(x, t)=\left\{\begin{array}{l}
T_{h}(x, t) \quad \text { if } \quad 0 \leq x<X_{h}(t) \\
\frac{\alpha \sqrt{2 \gamma_{h}}}{2 k\left(T_{f}\right) \sqrt{t}}\left(x-X_{h}(t)\right)+T_{f} \quad \text { if } \quad X_{h}(t) \leq x \leq X_{\infty}(t)
\end{array}\right.
$$

Lemma 8 The functions $\left.\tilde{T}_{h} \in C^{1}\left[0, X_{\infty}(t)\right)\right]$ satisfy $\left|\frac{\partial \tilde{T}_{h}}{\partial x}\right| \leq M$ on $\left[0, X_{\infty}(t)\right]$ for all $h>0$, $t>0$.

Proof. Let $t>0$ and $x \in\left[0, X_{\infty}(t)\right]$.

If $x \in\left[X_{h}(t), X_{\infty}(t)\right]$ then

$$
\left|\frac{\partial \tilde{T}_{h}(x, t)}{\partial x}\right|=\frac{\alpha \sqrt{2 \gamma_{\infty}}}{2 k\left(T_{f}\right) \sqrt{t}} .
$$

For otherwise, this is $x \in\left[0, X_{h}(t)\right)$ according to (7) and (13) we have

$$
\frac{\partial \tilde{T}_{h}}{\partial x}(x, t)=P^{\prime}\left(y_{h}^{2}\left(\frac{x}{X_{h}(t)}\right)\right) 2 y_{h}\left(\frac{x}{X_{h}(t)}\right) y_{h}^{\prime}\left(\frac{x}{X_{h}(t)}\right) \frac{1}{X_{h}(t)}
$$


Since $\frac{k}{s}$ is decreasing and $T_{m} \leq T_{h}(x, t) \leq T_{f}$, from (13) we have $y_{1} \leq y_{h}\left(\frac{x}{X_{h}(t)}\right) \leq y_{0}$, for all $h>0$. From (6) follows that

$$
\left|P^{\prime}\left(y_{h}^{2}\left(\frac{x}{X_{h}(t)}\right)\right)\right| \leq \frac{1}{2 \lambda y_{1} k_{m}}
$$

where $k_{m}=\min \left\{k(T), T_{m} \leq T \leq T_{f}\right\}$. Taking into account (29), (30), (53) and Lemma 6 we have

$$
\left|y_{h}^{\prime}\left(\frac{x}{X_{h}(t)}\right) \frac{1}{X_{h}(t)}\right| \leq \frac{1}{\sqrt{\pi t}\left[1-\operatorname{erf}\left(\frac{v_{1}}{\sqrt{2}}\right)\right]} .
$$

Then for $x \in\left[0, X_{h}(t)\right)$ results

$$
\left|\frac{\partial \tilde{T}_{h}(x, t)}{\partial x}\right| \leq \frac{y_{0}}{\lambda y_{1} k_{m} \sqrt{\pi t}\left[1-\operatorname{erf}\left(\frac{v_{1}}{\sqrt{2}}\right)\right]}
$$

Summaring, for all $h>0$ and $x \in\left[0, X_{\infty}(t)\right]$ we obtain

$$
\left|\frac{\partial \tilde{T}_{h}(x, t)}{\partial x}\right| \leq M=\max \left\{\frac{\alpha \sqrt{2 \gamma_{\infty}}}{2 k\left(T_{f}\right) \sqrt{t}}, \frac{y_{0}}{\lambda y_{1} k_{m} \sqrt{\pi t}\left[1-\operatorname{erf}\left(\frac{v_{1}}{\sqrt{2}}\right)\right]}\right\}
$$

and this precisely the assertion of the lemma.

Lemma 9 We have $\lim _{h \rightarrow+\infty} \tilde{T}_{h}(x, t)=T_{\infty}(x, t)$ for each $t>0$ and $x \in\left[0, X_{\infty}(t)\right]$.

Proof. Let $t>0$ and $x \in\left[0, X_{\infty}(t)\right)$. By Corollary 7 there exists $h_{0}=h_{0}(x)>0$ such that $x \in\left[0, X_{h}(t)\right]$ for all $h \geq h_{0}$. We consider $\tilde{T}_{h}(x, t)$ for $h \geq h_{0}$ we have

$$
\tilde{T}_{h}(x, t)=T_{h}(x, t)=P\left(\left(\varphi_{2 h}\left(\varphi_{1 h}^{-1}\left(x / X_{h}(t)\right)\right)\right)^{2}\right) .
$$

Taking into account Lemma 6, Corollary 7, (53) and (54) we obtain that the sequence $\left\{T_{h}(x, t)\right\}$ converges to $T_{\infty}(x, t)$. If $x=X_{\infty}(t)$ then $\tilde{T}_{h}\left(X_{\infty}(t), t\right)=T_{f}=T_{\infty}\left(X_{\infty}(t), t\right)$. $t>0$.

Hence, the sequence $\left\{\tilde{T}_{h}(x, t)\right\}$ converges to $T_{\infty}(x, t)$ pointwise on $\left[0, X_{\infty}(t)\right]$ for each

Theorem 10 For each $t>0$ we have the family of functions $\left\{\tilde{T}_{h}\right\}$ converges uniformly to $T_{\infty}$ for $h \longrightarrow+\infty$ on $\left[0, X_{\infty}(t)\right]$.

Proof. By Lemma 8 , for any $t>0$ the functions $\tilde{T}_{h}(x, t)$ are equicontinuous on $\left[0, X_{\infty}(t)\right]$ and from Lemma 9 converges pointwise to $T_{\infty}(x, t)$ for $h \longrightarrow+\infty$. Then, by Ascoli Arzela lemma we obtain their uniform convergence on $\left[0, X_{\infty}(t)\right]$. 


\section{Conclusions}

One phase nonlinear, one-dimensional Stefan problems for a semi-infinite material $x>0$, with phase change temperature $T_{f}$ has been considered with the assumption of a Storm's condition for the heat capacity and thermal conductivity and a convective condition at the fixed face. Existence and uniqueness of a similarity type solution has been obtained. Moreover, the convergence of this problem to problem with temperature condition at the fixed face when $h \rightarrow+\infty$ has been proved.

\section{Acknowledgment}

This paper has been partially sponsored by the Proyect PIP No. 112-200801-00534 "Inecuaciones Variacionales, Control Optimo y Problemas de Frontera Libre: teoría, análisis numérico y aplicaciones", from CONICET-UA, Rosario-Argentina and Proyect: "Ecuaciones a derivadas parciales, inecuaciones variacionales elípticas, problemas de frontera libre y de control óptimo y aplicaciones", Universidad Austral Rosario-Argentina.

\section{References}

1. Briozzo, A. C.; Natale, M. F.; Tarzia, D. A. Determination of unknown thermal coefficients for Storm's type materials through a phase-change process. Int. J. of Nonlinear Mechanics 1999, 34, 329-340.

2. Briozzo, A. C.; Natale, M. F. One-Dimensional Nonlinear Stefan Problems in Storm's Materials. Mathematics 2014, 2, 1-11; doi:10.3390/math2010001.

3. Carslaw, H. S.; Jaeger, J. C. Conduction of heat in solids, Clarendon Press, Oxford, 1965.

4. Hill, J. M.; Hart, V. G. The Stefan problem in nonlinear heat conduction. J. Appl. Math. Phys. (ZAMP) 1986, 37, 206-229.

5. Knight, J. H.; Philip, J. R. Exact solution in nonlinear diffusion. J. Engng Math. 1974, 8, 219-227.

6. Natale, M. F.; Tarzia, D. A. Explicit solutions to the two-phase Stefan problem for Storm's type materials. J. Phys. A:Math. Gen 2000, 37, 395-404.

7. Natale, M. F.; Tarzia, D. A. Explicit solution for a one-phase Stefan problem with temperature-dependent thermal conductivity. Bolletino della Unione Matematica Italiana, UMI 2006 , (8) 9-B, 79-99. 
8. Solomon, A. D.; Wilson, D. G.; Alexiades, V. Explicit solutions to phase change problems. Quart. Appl.Math. 1983, 51, 237-243.

9. Storm, M. L. Heat conduction in simple metals. J. Appl. Phys. 1951, 22, 940-951.

10. Tarzia, D. A., An inequality for the coefficient $\sigma$ of the free boundary $s(t)=2 \sigma \sqrt{t}$ of the Neumann solution for the two-phase Stefan problem. Quart. Appl. Math. 1981, 39, 491-497. 